\title{
Multi-photon microscope driven by novel green laser pump
}

Marti, Dominik; Djurhuus, Martin; Jensen, Ole Bjarlin; Andersen, Peter E.

\section{Published in:}

Proceedings of SPIE

Link to article, DOI:

$10.1117 / 12.2208586$

Publication date:

2016

Document Version

Publisher's PDF, also known as Version of record

Link back to DTU Orbit

Citation (APA):

Marti, D., Djurhuus, M., Jensen, O. B., \& Andersen, P. E. (2016). Multi-photon microscope driven by novel green laser pump. In A. Periasamy, P. T. C. So, \& K. König (Eds.), Proceedings of SPIE (Vol. 9712). [97121M] SPIE International Society for Optical Engineering. Proceedings of SPIE - The International Society for Optical Engineering https://doi.org/10.1117/12.2208586

\section{General rights}

Copyright and moral rights for the publications made accessible in the public portal are retained by the authors and/or other copyright owners and it is a condition of accessing publications that users recognise and abide by the legal requirements associated with these rights.

- Users may download and print one copy of any publication from the public portal for the purpose of private study or research.

- You may not further distribute the material or use it for any profit-making activity or commercial gain

- You may freely distribute the URL identifying the publication in the public portal 


\title{
Multi-photon microscope driven by novel green laser pump
}

\author{
Dominik Marti, Martin Djurhuus, Ole Bjarlin Jensen, and Peter E. Andersen \\ Technical University of Denmark, Department of Photonics Engineering, Frederiksborgvej 399, \\ 4000 Roskilde, Denmark
}

\begin{abstract}
Multi-photon microscopy is extensively used in research due to its superior possibilities when compared to other microscopy modalities. The technique also has the possibility to advance diagnostics in clinical applications, due to its capabilities complementing existing technology in a multimodal system. However, translation is hindered due to the high cost, high training demand and large footprint of a standard setup. We show in this article that minification of the setup, while also reducing cost and complexity, is indeed possible without compromising on image quality, by using a novel diode laser replacing the commonly used conventional solid state laser as the pump for the femtosecond system driving the imaging.
\end{abstract}

Keywords: Multi-Photon Microscopy, Diode Laser, Contrast, Image Quality

\section{INTRODUCTION}

Multi-Photon Microscopy (MPM) is an emerging tool in biological and clinical research. It is extensively used in research facilities, for label free imaging of cell function, ${ }^{1}$ for deep imaging of vasculature ${ }^{2}$ to visualize calcium dynamics in tissue, ${ }^{3}$ and also through endoscopes to stage or grade cancerous tissue, ${ }^{4}$ to name only a few. Its advantages include the use of endogenous fluorophores, a high penetration depth up to millimetres in biological media due to low scattering and absorption of the near infrared excitation light, low phototoxicity due to no or very low out-of-focus interaction of the light with the sample, easy separation of the excitation and emission light due to the big wavelength difference between the two, and intrinsic three-dimensional resolution due to the non-linear nature of the signal generation process. On the downside, MPM requires expensive equipment, the femtosecond laser system being the major cost factor, and is relatively slow, since the sample needs to be point-scanned to generate a $2 \mathrm{D}$ or $3 \mathrm{D}$ image.

MPM is mainly relying on the ultrashort pulses delivered by a Ti:Sapphire laser system. This system usually consists of a diode pumped, frequency doubled solid state laser emitting green light to pump the Ti:Sapphire crystal. This triple cascade of pumping lasers with other lasers is not only inefficient and needs water cooling due to the excess energy loss in the form of heat, but it also requires careful alignment of all the components, making it more difficult to handle. Due to the many components involved, the system is too expensive and also too bulky, hindering its application in other than research environments. To facilitate translation of the this technology to clinical applications, progress in minification, ease-of-use and cost efficiency are therefore needed.

The reason for using diode pumped solid state lasers as the pump for Ti:Sapphire lasers lies in their excellent performance with respect to output power and beam quality, the latter having a large influence on the pumping efficiency. Attempts are made to achieve the same properties with pure diode lasers. These lasers have the advantage of being more efficient than conventional solid state lasers, thus not requiring water cooling, while also being more compact. However, there is currently no pure diode solution that provides both a high output power and a good beam profile at the same time. Recently, however, it has been shown that second harmonic generation of a tapered laser diode output improves the beam profile drastically, due to the non-linear dependence of the conversion on the input intensity, effectively only converting the "nice" portions of the beam. ${ }^{5}$ Like that, a high power output can be achieved while still retaining a beam profile $\left(\mathrm{M}^{2} \approx 1.3\right)$ that suffices to pump a Ti:Sapphire laser $^{6}$ and to drive a multi-photon microscope. ${ }^{7}$ Using such a frequency doubled diode as the pump gets rid of

Further author information: (Send correspondence to Dominik Marti)

D.M.: E-mail: domar@fotonik.dtu.dk, Telephone: +45 46774568

P.E.A.: E-mail: peta@fotonik.dtu.dk, Telephone: +45 46774555

Multiphoton Microscopy in the Biomedical Sciences XVI, edited by Ammasi Periasamy, Peter T. C. So, Karsten König, Proc. of SPIE Vol. 9712, 97121M · @ 2016 SPIE · CCC code: 1605-7422/16/\$18 · doi: 10.1117/12.2208586 


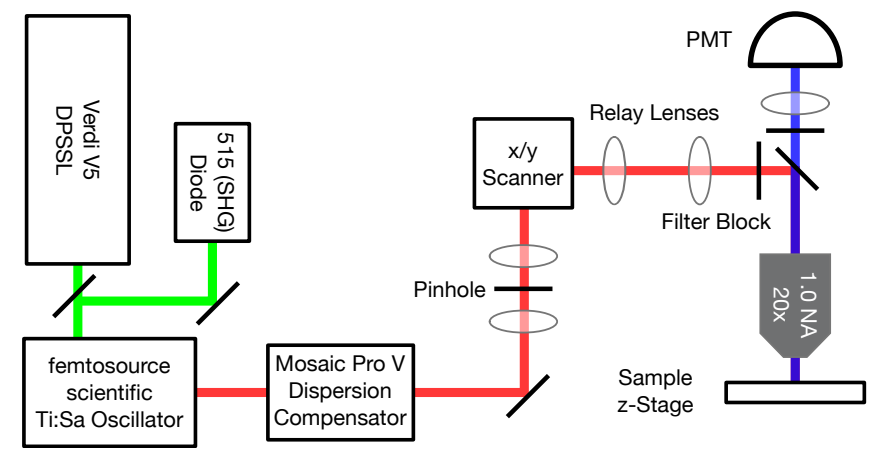

Figure 1. Schematic of the beam path. See the main text for details.

one of the intermediate conversion steps in the above mentioned cascade and makes water cooling superfluous, simplifying and minifying the setup and improving the efficiency of the whole system.

In our research, we are using both a conventional solid state laser (Verdi V5) as well as a frequency doubled tapered diode laser (TDL 515) as the pump for the same Ti:Sapphire laser, to compare the imaging performance of a custom built multi-photon microscope. We therefore record images of sample standards and compare them regarding signal-to-noise ratio and contrast, showing that the frequency doubled diode laser performs equally well as the conventional solid state laser.

\section{MATERIALS AND METHODS}

\subsection{Setup}

The laser system, shown in figure 1, consists of two switchable pump lasers; a frequency doubled diode pumped solid state laser (Verdi V5, Coherent, USA) emitting light at $532 \mathrm{~nm}$, and a frequency doubled tapered diode laser (TDL 515) built by DTU emitting light at $515 \mathrm{~nm}$. The pump can be chosen by swapping in and out one mirror, without any further alignment needed. The chosen pump laser beam is guided to a Ti:Sapphire oscillator (femtosource scientific s20, femtolasers, Austria) with $800 \mathrm{~nm}$ centre frequency, a bandwidth of $40 \mathrm{~nm}$ (measured with a S2000 spectrometer, Ocean Optics, USA), a repetition rate of $75 \mathrm{MHz}$ and a pulse duration of $20 \mathrm{fs}$ at the laser output. The beam is sent through a pre-compensating unit (mosaic pro v, femtolasers, Austria) to compensate for the dispersion further down the laser path, and through a spatial filter (KT130, Thorlabs, USA) using a $15.3 \mathrm{~mm}$ lens and a $10 \mu \mathrm{m}$ pinhole. The beam then propagates through a beam scanning unit (GVS012, Thorlabs, USA) and two relay lenses, before it gets transmitted through a long pass filter (FEL0700, Thorlabs, USA) and reflected of a dichroic mirror (HC720SP, AHF, Germany) to be guided to a 1.0 NA, 20× water dipping objective (XLUMPLFLN 20XW, Olympus) and focused on the sample. The fluorescent light is collected through the same objective, passes the dichroic mirror and a short-pass filter to remove any remaining infrared light, and is weakly focused onto a PMT (H7422, Hamamatsu, Japan). The sample is mounted on a motorized XYZ stage (MZS500-E Z-Axis Stage and MLS203-1 XY Stage, Thorlabs, USA). The whole setup is connected to a computer using a single data acquisition board (NI USB-6353, National Instruments, USA) and controlled by a custom made LabVIEW program. The sample used was a microscope slide with stained cells (FluoCells Prepared Slide \#2 BPAE cells, life technologies, USA). For all measurements, the laser power measured after the pinhole, before the beam scanner, was $9 \mathrm{~mW}$.

\subsection{Software}

We used Matlab to assess the quality of the images. To gauge the noisiness of the images, a routine was developed to split the image using fast Fourier transform into a low-frequency noise, a mid-frequency signal and a high-frequency noise part. These parts can then be compared to calculate a ratio between the total signal amplitude and the total noise amplitude, and these ratios (termed "amplitudes' ratio") are compared across images as a relative quality measure. We also used these processed images to calculate the peak signal-to-noise 

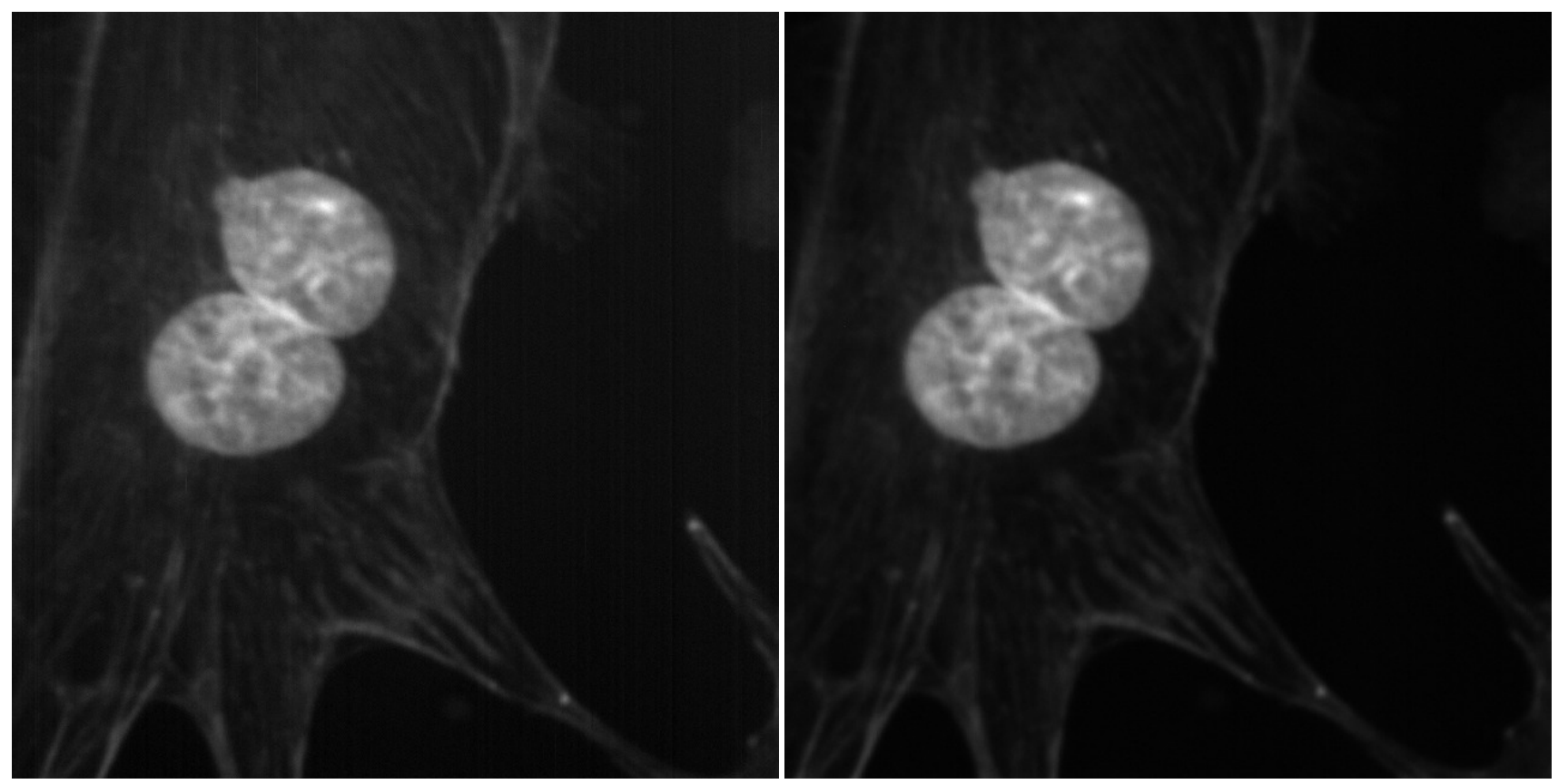

Figure 2. Multi-photon microscopy images of BPAE cells using the TDL 515 pump (left) and the Verdi V5 pump (right). The size of each image is $64 \mu \mathrm{m} \times 64 \mu \mathrm{m}$. The images were taken with the same incident power on the sample. Both images are shown with normalized intensity.

ratio (pSNR), using Matlab's psnr-function. The latter step can be seen as a mere conversion from the amplitudes' ratio into a more common value, a SNR, measured in decibels.

We additionally assessed perceived contrast using a routine described elsewhere. ${ }^{8}$ The perceived contrast is a measure for feature recognition by humans within the images. The routine we use was originally developed to assess contrast in every-day photographs, and whether it applies to microscopy images that normally have repetitive feature of specific sizes remains to be shown. Nevertheless, the obtained values can be compared across the different images taken using the two different pumps, keeping in mind that the values do not necessarily carry physical meaning, and it is not even clear whether a higher or lower value is desirable.

\section{RESULTS AND DISCUSSION}

To statistically assess the image quality using the two different pumps, we took two multi-photon microscopy images of FluoCells, one for each pump, at 31 different locations. Figure 2 shows one example of such an image pair taken with our system. Note that the system does not yet include filters to distinguish between different fluorophores, and the images are therefore only total fluorescence intensity maps. The quality measures described above for these two images are shown in table 1. We have chosen to show an extreme example out of the total 31 image pairs we have recorded, thus the quality measures do differ significantly. On the other hand, comparing the images themselves by eye suggests that there are small, but no striking differences in quality between the two.

Table 1. Image quality measures for the two images shown in figure 2.

\begin{tabular}{|l|c|c|l|}
\hline Pump & Amplitudes' Ratio & pSNR & Contrast \\
\hline \hline TDL 515 & 0.64 & $15.1 \mathrm{~dB}$ & 21.2 \\
\hline Verdi V5 & 1.13 & $17.9 \mathrm{~dB}$ & 30.1 \\
\hline
\end{tabular}

It can be seen that in this example the TDL 515 image (to the left) is noisier than the Verdi V5 image. This is also represented in both the amplitudes' ratio and the pSNR. However, as can be seen further down in 
the statistical assessment, the noise levels for the two pumps do in fact on average not differ significantly. The reason for the higher noise levels in one or the other image of the image pairs is yet unclear and currently under investigation.

The contrast measure also differs significantly for this pair: the TDL 515 image has a lower contrast. This can actually also be seen in the images themselves, where the cytoskeleton surrounding the nuclei in the TDL 515 image is brighter than it is in the Verdi V5 image. This leads to a lower intensity difference in the TDL 515 image than in the Verdi V5 image, which translates directly into lower contrast. There is also a contrast difference visible in the nuclei themselves. On the other hand, or maybe even because contrast is lower, the TDL 515 image seems to show more details of the cytoskeleton than the Verdi V5 image. From this example it can nicely be seen that a higher contrast does not necessarily relate to a better image or more information content, emphasising the above stated hypothesis that the contrast measure has to be taken with a grain of salt.

The images are each shown normalized, that is neither background signal nor dynamic range difference can be seen in this representation. In this case, the TDL 515 image had a higher background signal and a lower dynamic range, but this is not true for the whole dataset: we found that in 7 of the 31 image pairs, the Verdi V5 image shows a higher background, while in 21 the TDL 515 shows a higher background. In three cases, the background signal was comparable. Again, the reason for the difference in background signal is not yet clear.

Table 2. Averaged image quality measures for images taken with the the two pumps $(n=31)$.

\begin{tabular}{|l|c|c|l|}
\hline Pump & Amplitudes' Ratio & pSNR & Contrast \\
\hline \hline TDL 515 & $0.59 \pm 0.26$ & $15.7 \pm 4.0$ & $25.8 \pm 8.1$ \\
\hline Verdi V5 & $0.75 \pm 0.34$ & $16.9 \pm 4.2$ & $28.6 \pm 7.6$ \\
\hline
\end{tabular}

To more quantitatively measure the performance of the TDL 515 pump, we averaged the three quality measures over all 31 image pairs of the FluoCells, as shown in table 2. In this data, one can see that there is no significant difference between the two different pumps. This shows that using the TDL 515 pump, despite its slightly lower beam quality $\left(\mathrm{M}^{2} \approx 1.3\right)$, does not have a negative influence on the multi-photon microscopy image quality. We attribute this to the Ti:sapphire lasing in its preferred mode, independent of the pump beam shape, effectively rejecting pump light in a mode not overlapping with the laser mode, thus "cleaning" the beam.

\section{CONCLUSION}

We have shown that using a frequency doubled tapered diode laser can easily replace a conventional, frequency doubled diode pumped solid state laser in a common multi-photon microscopy setup. The use of the diode laser does not lead to degraded image quality, despite the fact that its beam quality is slightly inferior. The advantage of using the diode laser as the pump for the Ti:sapphire laser is its smaller footprint, better efficiency and lower cost when compared to a solid state laser. This can lead to a translation of the multi-photon technique from being purely research-centred to applications in clinics, where its excellent imaging properties in tissue can be exploited on a large scale, for example to screen for common diseases, thus benefiting both patients and health care costs.

\section{ACKNOWLEDGMENTS}

We thank Eli Peli (Massachusetts Eye and Ear) and Jonathan Nylk (University of St. Andrews) for providing us with the Matlab routines. We acknowledge financial support from the European Union project FAMOS (FP7 ICT, contract no. 317744).

\section{REFERENCES}

[1] Zipfel, W. R., Williams, R. M., Christie, R., Nikitin, A. Y., Hyman, B. T., and Webb, W. W., "Live tissue intrinsic emission microscopy using multiphoton-excited native fluorescence and second harmonic generation," Proceedings of the National Academy of Sciences 100, 7075-7080 (June 2003). 
[2] Kobat, D., Horton, N. G., and Xu, C., "In vivo two-photon microscopy to 1.6-mm depth in mouse cortex," Journal of Biomedical Optics 16(10), 106014-106014-4 (2011).

[3] Borile, G., de Mauro, C., Urbani, A., Alfieri, D., Pavone, F. S., and Mongillo, M., "Multispot multiphoton Ca2+ imaging in acute myocardial slices," Journal of Biomedical Optics 20(5), 051016-051016 (2014).

[4] Huland, D. M., Jain, M., Ouzounov, D. G., Robinson, B. D., Harya, D. S., Shevchuk, M. M., Singhal, P., Xu, C., and Tewari, A. K., "Multiphoton gradient index endoscopy for evaluation of diseased human prostatic tissue ex vivo," Journal of Biomedical Optics 19(11), 116011-116011 (2014).

[5] Jensen, O. B., Andersen, P. E., Sumpf, B., Hasler, K.-H., Erbert, G., and Petersen, P. M., "1.5 W green light generation by single-pass second harmonic generation of a single-frequency tapered diode laser," Optics Express 17, 6532 (Apr. 2009).

[6] Müller, A., Jensen, O. B., Unterhuber, A., Le, T., Stingl, A., Hasler, K.-H., Sumpf, B., Erbert, G., Andersen, P. E., and Petersen, P. M., "Frequency-doubled DBR-tapered diode laser for direct pumping of Ti:sapphire lasers generating sub-20 fs pulses," Optics Express 19, 12156 (June 2011).

[7] König, K., Andersen, P. E., Le, T., and Breunig, H. G., "Multiphoton imaging with a novel compact diode-pumped Ti:sapphire oscillator," Microscopy Research and Technique 78, 1154-1158 (Dec. 2015).

[8] Haun, A. M. and Peli, E., "Perceived contrast in complex images," Journal of Vision 13 (Nov. 2013). 